\title{
A Study on Integrated Construction of Luzhou Comprehensive Transportation Hub*
}

\author{
Peng Xu, Li Yan \\ Southwest Medical University, Luzhou, Sichuan, China
}

\begin{abstract}
The target of integrated construction of comprehensive transportation hub is to integrate the traffic resources, achieve butt joint of pan-regional transportation mode, and finally realize the "seamless connection" of the goods and "zero transfer" of the passenger traffic. Combining practice of Luzhou, taking the advanced experience of foreign and domestic cities for reference, the paper pointed out that for the sake of constructing a comprehensive transportation hub, we need to build the traffic integration on the basis of the efficiency. Besides, the paper proposed the strategies for construction.
\end{abstract}

Keywords: comprehensive transportation hub, integration, construction strategies

Construction of comprehensive transportation hub is the premise and basic condition for regional economic development. With the development of market economy, the contradiction of supply-demand imbalance of traffic resources is becoming increasingly prominent. Based on the target of improving efficiency, the construction of integrated comprehensive transportation hub is an important approach to relieving traffic jam and developing regional economy. Luzhou is located in the vital communication line of Southern Sichuan as well as the intersection of Yangtze River and Tuojiang River. Relying on the importance of geographical position and the convenience of the Yangtze River golden channel, Luzhou strives to build a comprehensive transportation hub. The traffic infrastructure construction is tending towards perfection, while the deficiencies in management and improvement are becoming increasingly prominent, thus the integration of comprehensive transportation hub should be constructed from the perspective of efficiency and butt joint of pan-regional transportation resources should be achieved to promote the economic development.

\section{Current Situation of Construction of Comprehensive Transportation Hub in Luzhou} \section{Situation of Traffic Infrastructure Construction of Luzhou}

Luzhou is located in the southeast of Sichuan Province, the joint place of Sichuan, Yunnan, Guizhou, and Chongqing, the intersection of the Yangtze River and Tuojiang River, and the transitional zone from southern margin of Sichuan Basin to the Yunnan-Guizhou Plateau, so it is a regional secondary comprehensive

\footnotetext{
* The paper is supported by western transportation strategy and regional development research center project: the research on coordination mechanism of southern Sichuan economic zone port group and port-surrounding industry development (No. XJQ008).

Peng Xu, master, lecturer, School of Humanities and Management Sciences, Southwest Medical University, Luzhou, Sichuan, China; research field: logistics management.

Corresponding author: Li Yan, master, associate professor, School of Humanities and Management Sciences, Southwest Medical University, Luzhou, Sichuan, China; research field: management.
} 
transportation hub for Sichuan to highlight southing and strengthen easting. Luzhou Municipal Government is making efforts to build a comprehensive transportation hub which radiates to the surrounding areas and promotes the regional economic growth.

On the whole, the traffic infrastructure construction of Luzhou basically has the framework of regional integrated transportation hub. Relying on the Luzhou Port, Luzhou City is building a three-dimensional traffic network with waterway, highway, railway, and aviation as the focuses, and the target is to build Luzhou into a regional integrated transportation hub of Sichuan that highlights southing, leads to the river and sea, and connects Southeast Asia with perfect function and distinct gradation. At present, Luzhou has basically completed the highway network of "one ring, seven radiations, one transverse line" with the main urban area as the center, the railway network of "three vertical lines and four horizontal lines", the water transportation system of "one horizontal line, two vertical lines, five harbor districts", and the important feeder airport in the joint place of Sichuan, Yunnan, Guizhou, and Chongqing.

\section{Deficiencies in Construction of Luzhou Comprehensive Transportation Hub}

Decentralized management and poor coordination. Various modes of transportation are the foundation to realize multimodal transport, but the high efficiency of multimodal transport is not reflected adequately, for which the primary problem lies in that relevant government administrative departments do things in their own way, and a coordination department for unified management is absent. The various modes of transportation are managed by different departments, thus the planning, decision-making, and management are considered from the perspective of interests of the respective departments, and global consciousness is lacking. The strengths of the various modes of transportation are dispersed, so abundant financing cannot be available. Compared with the comprehensive financing credit of overall transport, the channel is relatively narrow. This is unfavourable for the long-term development of the construction of comprehensive transportation hub.

Relatively high logistics cost and low efficiency. The infrastructure construction is of large-scale cooperation, but unified planning and construction is lacking, and the internal integration cannot be achieved in a timely manner. Meanwhile, the integration efficiency of overall mechanism of multimodal transport is not high enough, the informationization degree is low, and the logistics service system is not perfect, resulting in relatively high logistics costs and low efficiency. The key of construction of integrated comprehensive transportation hub not just lies in the comprehensive laying of the infrastructure, more importantly, the use efficiency of traffic resources can only be improved with sound relevant services, while efficiency is one of the most important indicators measuring the comprehensive transportation capacity. Insufficient information sharing and unclear management give rise to longer time consumption in communication media transfer and high unloaded ratio of transport machine, which makes the high cost of logistics, and is a manifestation of insufficient transportation resources development and utilization.

Absence of government relevant laws and regulations and policy guidance. Freight transport should select proper transport mode based on the quantity, quality, and type of the goods. Since various modes of transport coexist in the comprehensive transportation hub, structural contradiction of the various modes of transport may appear. The same thing goes for the stream of people. If the mode of passenger transport can be divided into private car travel and public transport travel, then vigorously promoting the public transport is essential, which can not only achieve the purpose of environmental protection and emission reduction, but also facilitate the unrestricted flow of people and relieve traffic pressure. Whether solving the structural 
contradiction caused by coexistence of multiple transport modes or encouraging the implementation of public transport mode, the guide of government is a must. However, in Luzhou, in the comprehensive transportation hub, government's work is relatively lacking. Without guidance of relevant laws and regulations and relevant political leanings, the transportation resources are completely market-oriented allocated, which may make the resources develop disorderly.

Absence of humanized consideration. Priority policy for public transport is scarce, the transfer and parking are not convenient, the public transport environment is not elegant enough, and it is not convenient enough for the public to use the public transport for travel, all of which cause a large number of people to choose to travel by private cars. The target of planning and development of transportation system should be to improve the quality of life and comfort level of the public, meanwhile, the environmental protection should also be included into the consideration scope of the construction. Nevertheless, the current traffic construction in Luzhou vigorously promotes the improvement of infrastructure, while consideration in details is lacking, humanization design for transport services is insufficient, traffic environment protection and sustainable development are not paid attention to, and environmental influence, greening, and ecological protection are almost ignored in traffic project evaluation.

\section{Strategies for Construction of Integration of Luzhou High Efficient Comprehensive Transportation Hub}

\section{Reinforcement of Government Guidance}

Establishment of "integrity" consciousness for the comprehensive transportation hub. From the perspective of construction concept, the government needs to break the constraint of traditional concept. The construction of comprehensive transportation hub should not focus on Luzhou City. The pattern of constructing integrated transportation should be placed in the region as a whole, i.e., transforming the development of single territory into the development of the whole region. The unique geographical location has brought development opportunities for Luzhou, so the construction of a comprehensive transportation hub is imperative. If the construction is limited to the inner traffic of single area, while regional "integrity" awareness is lacking, the construction pattern will be low-end and narrow, which is not beneficial to the long-term development. Therefore, in terms of ideology, the government should have a regional "integrity" consciousness and establish a sense of general situation for overall development of regional transport, in order to achieve a win-win situation.

Establishment of integrated management system. In our country, various modes of transportation have their corresponding parallel administrative departments. This kind of fragmented management approach may cause the departments to fight for their respective interests or uncoordinated, unified situation in the process of construction of integration of high efficient comprehensive transportation hub, thus the modes of transport that may be effectively connected at first appear separation from each other in some links, resulting in that the various modes of transport cannot be perfectly connected in a real sense in terms of standards and rules, leading to low efficiency and high cost. To establish an integrated management system, not only the coordination of various traffic management departments is needed, but also the coordination of relevant government agencies is a necessity, so that the management functions can be further concentrated, the management by multiple departments or intersecting management can be avoided and a more harmonious integrated transportation management system can be established to adapt to the specific demands of integrated transportation 
management. So far, the transportation management departments in some provinces and cities of our country have established informal macro-transportation management system, which has become a reference for Luzhou to establish integrated management and coordination of various modes of transport in construction of comprehensive transportation hub. The emergence of such approach is undoubtedly the prelude to perfect the integrated transportation management system.

Improvement of the laws and regulations and preferential policies. To construct the high efficiency and integration of the comprehensive transportation hub, first of all, impeccable laws and regulations are needed as the specification of management. In the specification of integrated traffic relevant laws and regulations, strict regulation and supervision should be carried out on management, safe operation, and efficiency of transportation. Besides, strict inspection should be conducted on the operation, management, services, security, and even environmental protection of logistics enterprises according to laws and regulations, so that the overall traffic transport and development of related industries will be more efficient and orderly. Impeccable laws and regulations are the necessary conditions for Luzhou to build a highly efficient integrated transportation hub and realize its sustainable development of integration. At the same time, the policy guidance should be strengthened and inclined to the development of public transport, so that the public transport can become the first choice for people to travel. To relieve the traffic congestion in time interval, the construction of public transport infrastructure needs to be strengthened. In order to achieve fast, convenient, and economic public transportation, the government subsidies to the public transport can be properly offered, and the special planning for public transportation routes can be made.

\section{Integration of Regional Transportation Resources}

To construct the high efficiency and integration of comprehensive transportation hub, the construction of transportation must be blended into the regions, which requires the traffic resources of Luzhou to achieve butt joint with the surrounding urban circle, so that Luzhou can play the role of hub in a real sense. The regional transportation integration needs to establish multi-layered regional transportation coordination, including docking of infrastructure laying, pan-regional coordination of government agencies, cooperation of traffic management, and demand convergence of cross-regional transportation resources. In the process of construction of regional transportation integration, internally speaking, the traffic planning and layout of Luzhou need to be coordinated with the entire city and even the overall region, and the general situation and perspectiveness of traffic development should be kept; externally speaking, Luzhou needs to strengthen its external relatedness, and maintains connectivity with its surrounding urban agglomeration. Therefore, when the transportation resources sharing with urban agglomeration is achieved, Luzhou's role as comprehensive transportation hub will be highlighted, the integration of regional transportation will be better promoted, and the city can be enabled to achieve coordinated and sustainable development of traffic, economy, and environment.

\section{Construction of Integrated Information Platform}

The construction of comprehensive information platform refers to using advanced information technology to build high efficient information service system, so as to keep the costs down, reduce the transfer time, distribute goods intelligently, and achieve coordination of transportation and services. The platform mainly includes the information management system of traffic affairs, traffic operation management system, traffic database platform, traffic service information system, etc. The construction of integrated information platform needs to rely on the computer technology and create an integrated open network system of transportation 
services. In terms of overall information construction, the cargo intelligent distribution system will be created, then cargo transportation can be divided according to the category, quantity, dimension, volume, and flow direction, so more reasonable modes of transportation can be selected to reduce costs; in terms of logistics supporting information service, to build the integrated information platform, in addition to the direct construction of traffic information resources, impeccable service information of logistics supporting facilities is needed, such as logistics park service, storage information of dock and goods yard, and so on; in terms of freight transport, a freight supply-demand platform should be established to provide more comprehensive and extensive supply and demand information of logistics and transportation, so that the unloaded ratio can be reduced and sustainable development of highway transportation industry can be promoted; in terms of passenger transport, a WeChat \& Internet comprehensive passenger platform should be constructed, and "one ticket" service should be achieved. In doing so, the transfer passengers can save time and the travel will be more convenient.

\section{Promotion of Service Level of Professional Logistics}

The construction of integration of high efficient comprehensive transportation hub needs impeccable traffic infrastructure construction and high-end information platform; in addition, supporting professional logistics services are also needed. The construction of third party logistics should be vigorously promoted, since logistics service level is an important way to lower the logistics cost. Luzhou has built a number of logistics parks, which provide convenience for the flow of goods within the region and achieve a decline in the cost of logistics to a certain extent. Modern logistics services, especially the third party logistics services, mainly include warehousing, transportation, and other integrated services. The current logistics service of Luzhou can by and large bear the basic warehousing and transportation services, but other integrated services have not been effectively carried out, such as the warehouse financing. To complete logistics services of high quality, in addition to thorough information, upgrade of the service function is imperative. Hence, we should enhance the service capabilities of third party logistics, improve the service functions, and expand the network of logistics services. At the same time, the concept of logistics service also needs to be further promoted. We should regard the customers as the core, not only locate the logistics services in warehousing and transportation, but also increase the value-added service with promotion as the core. Besides, professional staff should be utilized for reasonable arrangement to eliminate unnecessary duplication of work, so that the time consumption can be reduced to the greatest extent and customer satisfaction can be enhanced.

\section{Conclusions}

The construction of integration of Luzhou comprehensive transportation hub from the perspective of efficiency is the inevitable course for Luzhou to integrate traffic resources and develop the logistics relevant industries. The construction of integrated comprehensive transportation hub in Luzhou can promote the industrial structure, develop the logistics relevant industries, and drive the development of regional economy; meanwhile, the development of regional economy can allow Luzhou to have the ability and strength to further develop its transportation industry, so the two complement each other. To construct the integration of transportation based on efficiency, the regional transport resources need to be integrated and humanization and environmental protection should be regarded as the construction goals, which is also of deep meanings for enhancing public transport comfort level and constructing urban culture. 


\section{References}

Bo, B. (2012). Regional comprehensive transportation integration-Operation mechanism and efficiency (pp. 95-117). Beijing: Economic Management Press.

Chen, M., \& Wang, J. G. (2012). Integration and intelligence-Revelations of German integrated transportation system and highway intelligent traffic. China Highway, 2012(02), 118-121.

Jiao, C. Z. (2013). Integrated construction management practice of large scale comprehensive transportation hub. Guangdong Architecture Civil Engineering, 40(3), 51-54.

Li, Z. X. (2004). Discussion about the mutuality of traffic integration construction in the three gorges region. Chongqing Architecture, 2(2), 16-18.

Lian, Y. P., \& Yang, J. Q. (2009). An introduction to integrative traffic transportation (pp. 1-14). Chengdu: Southwest Jiao Tong University Press.

Sun, X. W. (2008). Key path to promote the construction of traffic integration. China Water Transport, 8(6), 230-231.

Wang, Q, Y. (2002). Construction and development of comprehensive transportation system. Journal of Transportation Systems Engineering and Information Technology, 2(03), 56-60.

Zhang, X. Y., \& Zhang, G. W. (2001). Virtual enterprise organization negotiation mode of comprehensive transportation hub. Systems Engineering Theory Methodology Applications, 10(3), 244-247.

Zhang, Z. L. (2011). Study on speeding up the construction of traffic integration in Harbin. China Urban Economy, 22(14), 19-20.

Zhu, B. J. (2005). Countermeasures and research of traffic integration development in Shenyang economic zone. Master thesis, Northeastern University. 